This document is the Accepted Manuscript version of a Published Work that appeared in final form in ACS Nano, copyright (C) American Chemical Society after peer review and technical editing by the publisher.

To access the final edited and published work see https://pubs.acs.org/articlesonrequest/AOR-jmvnuAmdHrA7MKvf3uMs. 


\title{
Implanting Germanium into Graphene
}

\author{
Mukesh Tripathi, ${ }^{* \dagger}$ Alexander Markevich,,+ Roman Böttger, $₫$ Stefan Facsko, \\ Elena Besley, ${ }^{\ddagger}$ Jani Kotakoski, ${ }^{\dagger}$ and Toma Susi ${ }^{*, \dagger}$ \\ $\dagger$ University of Vienna, Faculty of Physics, 1090 Vienna, Austria \\ $\ddagger$ University of Nottingham, School of Chemistry, NG7 2RD Nottingham, UK \\ \Helmholtz-Zentrum Dresden-Rossendorf, Institute of Ion Beam Physics and Materials \\ Research, 01314 Dresden, Germany \\ E-mail: mukesh.tripathi@univie.ac.at; toma.susi@univie.ac.at
}

\begin{abstract}
Incorporating heteroatoms into the graphene lattice may be used to tailor its electronic, mechanical and chemical properties, although directly observed substitutions have thus far been limited to incidental Si impurities and $\mathrm{P}, \mathrm{N}$ and $\mathrm{B}$ dopants introduced using low-energy ion implantation. We present here the heaviest impurity to date, namely ${ }^{74} \mathrm{Ge}^{+}$ions implanted into monolayer graphene. Although sample contamination remains an issue, atomic resolution scanning transmission electron microscopy imaging and quantitative image simulations show that Ge can either directly substitute single atoms, bonding to three carbon neighbors in a buckled out-of-plane configuration, or occupy an in-plane position in a divacancy. First principles molecular dynamics provides further atomistic insight into the implantation process, revealing a strong chemical effect that enables implantation below the graphene displacement threshold energy. Our results demonstrate that heavy atoms can be implanted into the graphene lattice, pointing a way towards advanced applications such as single-atom catalysis with graphene as the template.
\end{abstract}


Keywords: ion implantation, heteroatom doping, scanning transmission electron microscopy, electron spectroscopy, molecular dynamics

Graphene $^{1}$ is an atomically thin single layer of hexagonally bound carbon with remarkable thermal, ${ }^{2}$ mechanical, ${ }^{3}$ and electrical properties. ${ }^{4}$ These outstanding properties combined with large-scale fabrication of high-quality single-crystal graphene films ${ }^{5,6}$ make it an ideal material for practical applications ranging from composites ${ }^{7}$ to optoelectronics. ${ }^{8}$ However, for many purposes, the atomic structure of graphene is chemically too inert, or the electronic structure needs to be modified. One way to achieve this is to incorporate heteroatoms as impurities into the structure, commonly introduced via a suitable chemical precursor during synthesis. ${ }^{9}$ However, due to a lack of control over their bonding and contamination by different chemical remnants, the quality of the produced structures may be limited, ${ }^{10,11}$ nor do all elements have easily available precursors. An alternative is to use ion implantation.

Ion implantation is a mature technique to manipulate materials that offers great flexibility: a plethora of ion species, a wide range of implantation energies, and control over the dopant concentration through the ion fluence. It is widely used by the modern semiconductor industry to introduce controlled concentrations of $p$ and $n$-type dopants at precise depths below crystal surfaces. Recently, single ions in semiconductors such as silicon, germanium and arsenic have attracted interest for the development of quantum computers. ${ }^{12}$ However, ion implantation is quite challenging in the case of two-dimensional (2D) materials such as graphene since only a narrow energy window will allow implantation - high enough to remove one or more target atoms yet low enough to stop the ion within the atomically thin structure. ${ }^{13}$ Moreover, since adatoms on 2D crystals tend to be mobile ${ }^{14}$ and there are typically no available interstitial sites, the implanted ion needs to form covalent bonds with the under-coordinated atoms of the vacancy to reach a stable configuration. After early work on carbon nanotubes ${ }^{15,16}$ the direct substitutional doping of graphene ${ }^{17,18}$ and transition metal dichalcogenides ${ }^{19}$ has been achieved using low-energy ion implantation, but large-scale controlled dopant incorporation into 2D materials remains an important challenge. 
Germanium $(\mathrm{Ge})$ is a structural and electronic analogue to silicon ( $\mathrm{Si})$, but significantly heavier (atomic number 32 as compared to 14) and larger (covalent atomic radius of 122 $\mathrm{pm}$ as compared to $111 \mathrm{pm}$ for $\mathrm{Si}$ and $77 \mathrm{pm}$ for $\mathrm{C}$ ). This raises the question whether it could also be incorporated into graphene similar to the lighter boron and nitrogen, ${ }^{17,20}$ the often observed silicon, ${ }^{21-23}$ and the recently implanted phosphorus, ${ }^{24}$ all of which are able to directly substitute for single $\mathrm{C}$ atoms. The substitutional doping of graphene with Ge is expected to be enhanced by co-doping with $\mathrm{N},{ }^{25}$ and spectroscopically characterized $\mathrm{Ge} / \mathrm{N}$ doped multiwalled carbon nanotubes recently showed excellent catalytic performance. ${ }^{26}$ Due to its greater size, a significant increase of bond length resulting in threefold Ge buckling out of the graphene plane is predicted. ${ }^{27}$ Its isovalence with $\mathrm{Si}$ anticipates ${ }^{22}$ another possible bonding: a (nearly ${ }^{28}$ ) planar fourfold substitution in a double vacancy. Based on density functional theory (DFT) calculations, doping graphene with germanium can open and control the band gap depending on concentration. ${ }^{27,29}$ However, no direct evidence for the stability of direct lattice substitutions has yet been reported.

\section{Results and Discussion}

We have successfully incorporated Ge into the graphene lattice using low-energy ion implantation. Although even our best implanted samples remain heavily contaminated, ${ }^{24}$ atomic resolution scanning transmission electron microscopy (STEM) shows that Ge can directly substitute for single atoms, bonding to three carbon neighbors in a buckled out-of-plane configuration as predicted by DFT. The chemical nature of the dopants is confirmed through quantitative image simulations and energy dispersive x-ray spectroscopy (EDX), whereas electron energy loss spectroscopy (EELS) is hardly sensitive to Ge due to spectral overlap with the much more intense graphene $\pi+\sigma$ plasmon response. ${ }^{30}$ We describe the atomic-level details of the implantation process through ab initio molecular dynamics (MD) simulations, which reveal a strong chemical effect that reduces the required implantation energy for Ge 
as compared to purely kinematic arguments. This is experimentally verified by successful implantation below the carbon displacement threshold energy in graphene. Our results show that elements beyond the third period can be directly implanted into graphene, expanding the versatility of ion irradiation as a tool for engineering graphene.

Contamination introduced during implantation degrades sample quality and makes characterization difficult. Like in previous P-implanted samples,${ }^{24}$ most of the graphene surface is covered by a thick layer of contamination (a medium angle annular dark field, MAADF, image is presented in Fig. 1a), despite laser treatment both before and during the implantation (Methods). The degree of contamination was however not entirely consistent: in some samples we were not able to find any clean lattice despite extensive searching, whereas in others this was rare, but included regions with implanted impurity atoms. Due to this variation, which was greater than in earlier P-implanted graphene ${ }^{24}$ we cannot exclude the possibility that our laser alignment varied between the different experiments.

Most of our data is from the cleanest sample that was irradiated at $20 \mathrm{eV}$. We did also find one incorporated impurity atom in a sample irradiated at $15 \mathrm{eV}$, but found no clean lattice in the sample irradiated at $25 \mathrm{eV}$. This lack of statistics from different ion energies prevents us from deducing the ideal implantation energy from the experimental data-in total, we found only seven Ge impurities directly in the lattice (roughly $1500 \mathrm{~nm}^{2}$ of clean lattice was analyzed; with our ion fluence of $1 \mathrm{ion} / \mathrm{nm}^{2}$, this yields an implantation efficiency of just $0.5 \%$ ). An example of a relatively clean sample area is presented in Fig. 1b, illustrating how most clean areas do not contain any Ge atoms (or other defects). Examples of heavy impurities found in the contamination as well as incorporated into the lattice are presented in Figs. 1c,d.

EELS is usually the best tool for establishing chemical identities and bonding at the atomic level. ${ }^{17,20,24}$ However, Ge impurities in graphene present a particular challenge: the weak Ge $L$-edge is located at $\sim 1150 \mathrm{eV}$, beyond the range of our spectrometer, whereas the $M_{4,5}$-edge, located at $\sim 32 \mathrm{eV}$, falls under the much more intense graphene $\pi+\sigma$ plasmon 

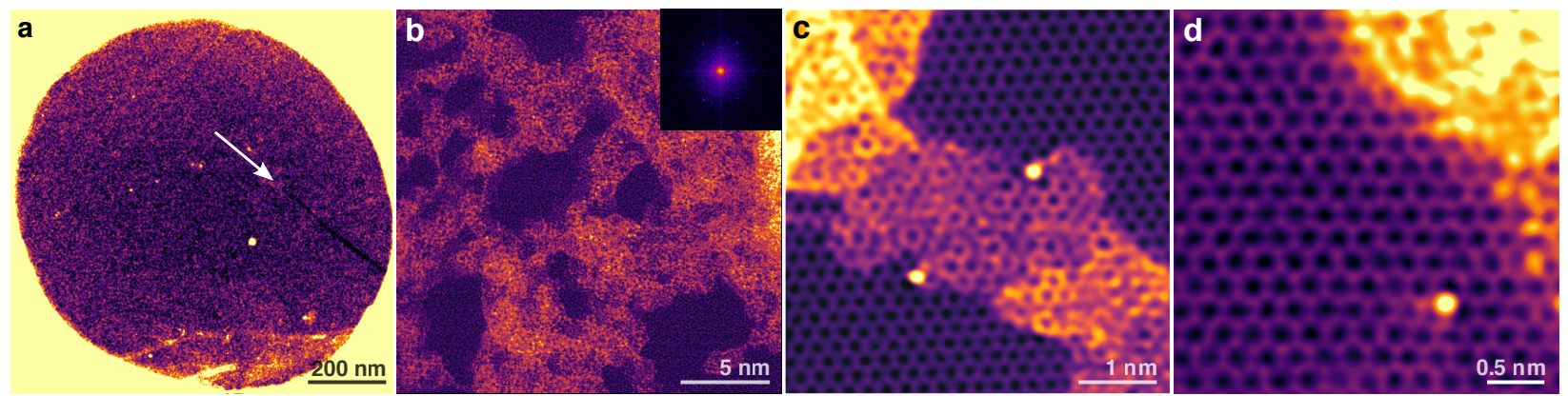

Figure 1: Overviews of a graphene sample implanted with $20 \mathrm{eV}{ }^{74} \mathrm{Ge}^{+}$ions (MAADF/STEM, $1024 \times 1024 \mathrm{px}$ ). (a) Raw image of heavily contaminated graphene suspended over a Quantifoil hole after ion implantation, with the arrow indicating a narrow cleaner region. (b) Clean monolayer graphene areas some tens of $\mathrm{nm}^{2}$ in size were found in this region. The fast Fourier transform (FFT) of the inset shows the hexagonal structure of the graphene lattice. (c) Two Ge atoms bonded at the edge of overlaying graphitized contamination. (d) A single Ge atom incorporated into the graphene lattice. Panels b-d have been processed with double Gaussian filtering to reduce the probe tail effect. ${ }^{31}$

(which is further influenced by nearby contamination ${ }^{32,33}$ ). As a consequence, in the EEL spectrum maps of Figs. 2b,c, it is not possible to distinguish the signal of the Ge atoms from the background of the plasmon tail, even though the impurities are clearly visible in the simultaneously acquired high angle annular dark field (HAADF) image (Fig. 2a). Nonetheless, as shown in Fig. 2d, a high signal-to-noise spot spectrum recorded over a single Ge substitution shows an apparent splitting of the plasmon peak as well as additional intensity compared to pristine graphene at higher energy losses. Although subtracting a scaled reference spectrum of thin-film bulk Ge (Gatan EELS Atlas ${ }^{34}$ ) with an intense plasmon component centered at $\sim 17 \mathrm{eV}$ in addition to the $M_{4,5}$-edge starting at $29 \mathrm{eV}$ (Ref. 35) can partially account for both features, further studies with monochromated EELS ${ }^{36}$ seem warranted. However, as shown in Fig. 2e, it is much easier to detect the spectroscopic signature of Ge using EDX, which should in a suitable instrument be feasible down to the single-atom level. ${ }^{37}$

Due to the atomic number-dependent scattering contrast in annular dark field images, ${ }^{31}$ we can turn to quantitative image simulations to verify the identity of the observed impurities. ${ }^{38}$ The atomic resolution STEM images shown in Fig. 3 give two examples of an 

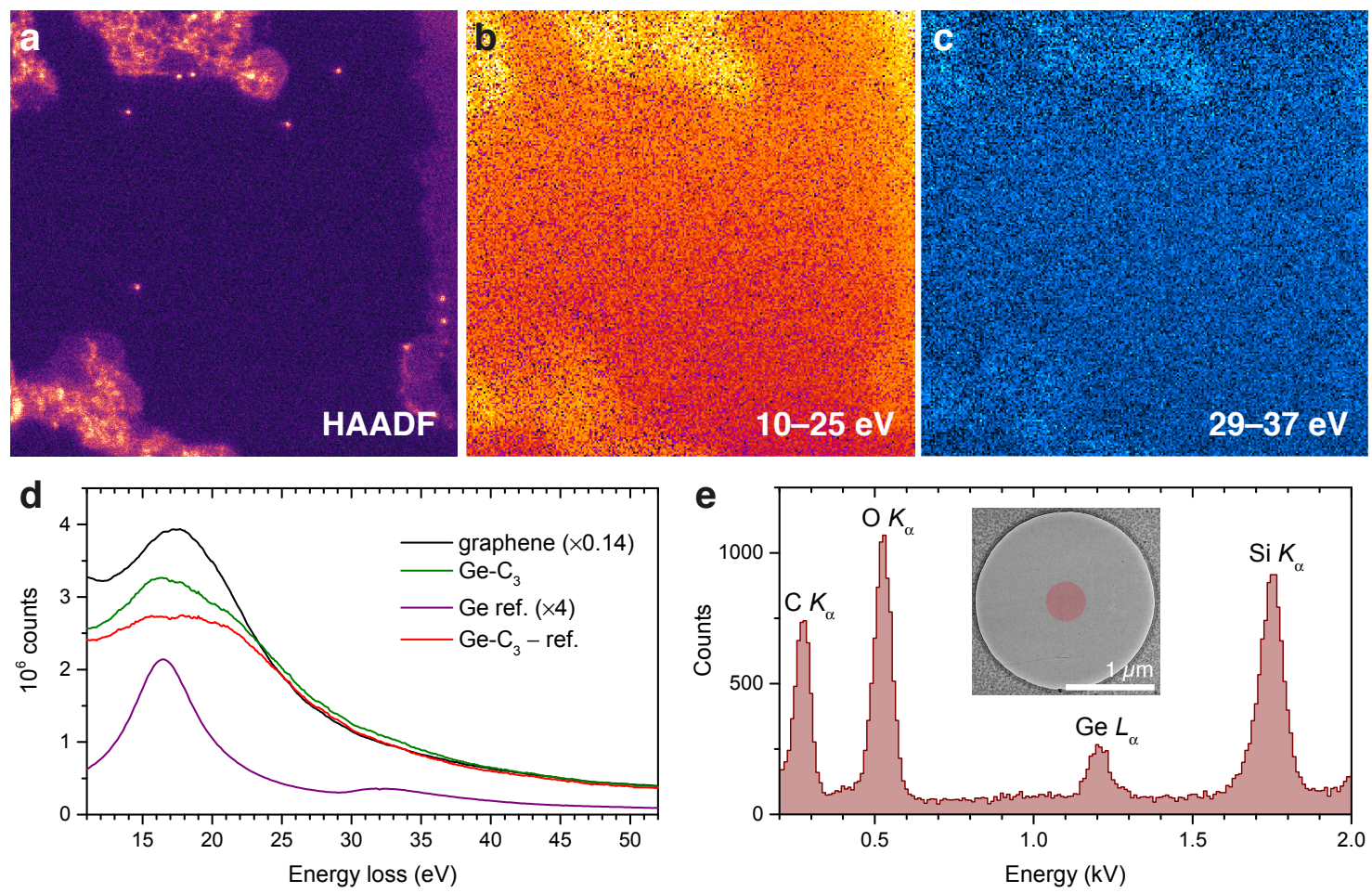

Figure 2: Spectroscopic characterization of implanted germanium. HAADF/STEM image of a region with multiple Ge atoms found in the lattice and in the contamination layer. (b) Background-subtracted EELS spectrum map integrated over the $\pi+\sigma$ plasmon response. (c) As in b, but integrated over the Ge $M_{4,5}$-edge. (d) Spot EELS spectra measured over pristine graphene and over a single Ge substitution $\left(\mathrm{Ge}^{-\mathrm{C}_{3}}\right.$, shorter exposure), along with a thin-film Ge reference (Ge ref.) and their difference $\left(\mathrm{Ge}^{-} \mathrm{C}_{3}-\mathrm{ref}\right.$.). (e) EDX spectrum with the approximate beam size indicated by the colored red area in the inset bright-field image of graphene suspended over a hole in the carbon support foil.

individual heavy impurity within the graphene lattice. To reduce the influence of the electron beam probe tails, these MAADF images have been processed using a double Gaussian filter. ${ }^{31}$ Scattering at the impurity is extremely intense even to the MAADF detector, making it difficult to distinguish the local structure. However, simulated images of threefold $\left(\mathrm{Ge}^{-\mathrm{C}_{3}}\right)$ and fourfold $\left(\mathrm{Ge}_{\mathrm{C}} \mathrm{C}_{4}\right)$ germanium substitutions (Fig. 3) agree well with the filtered images. From HAADF images (that avoid nonlinear scattering effects present in MAADF images), we measure the ratio of the intensity of the impurity atom to that of carbon atoms distant from it as $21.5 \pm 0.9$ for $\mathrm{Ge}_{-} \mathrm{C}_{3}$ and $21.7 \pm 1.0$ for $\mathrm{Ge}_{-} \mathrm{C}_{4}$, with simulated ratios respectively of 21.8 and 22.5. This corresponds to a $\mathrm{Ge} / \mathrm{C}$ contrast proportional to $\mathrm{Z}^{1.86}$, and the agreement confirms the chemical identity of the impurities. Our DFT simulation reproduces the 


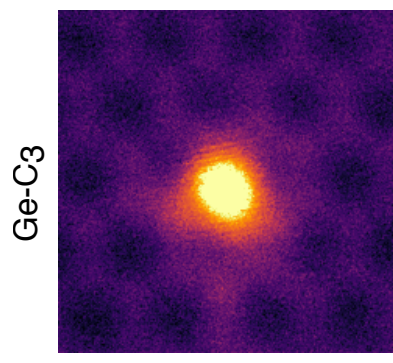

raw

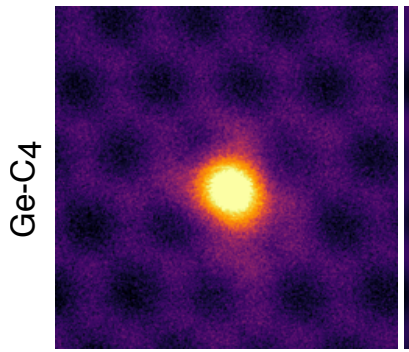

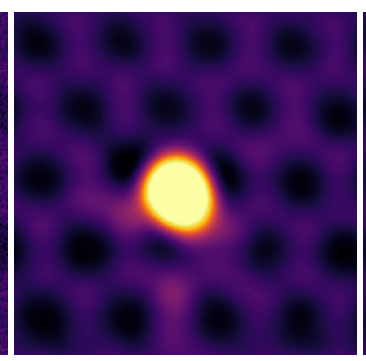

filtered

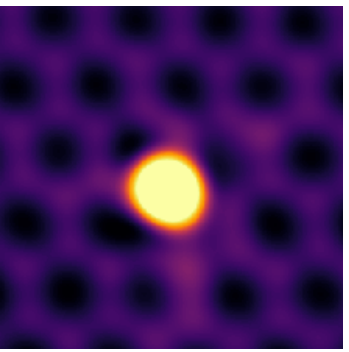

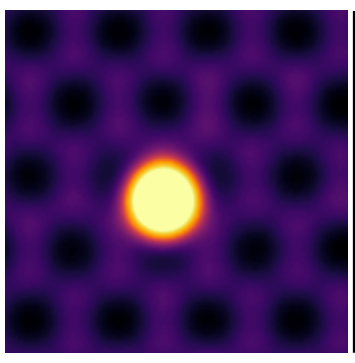

simulated

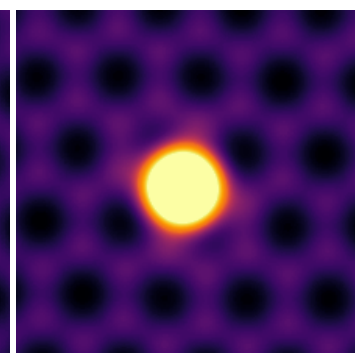

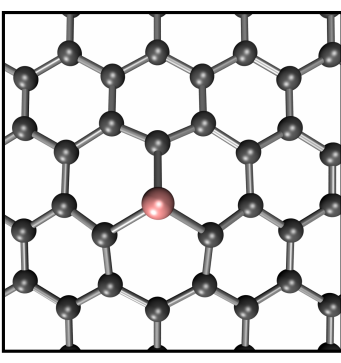

model (top)

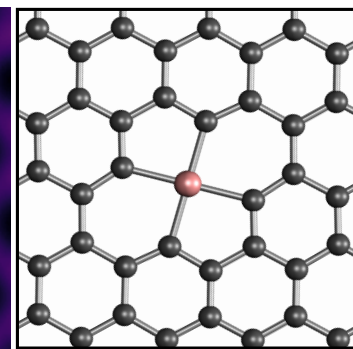

(side)

Figure 3: Germanium substitutions in graphene (top: threefold single atom substitution, $\mathrm{Ge}_{-} \mathrm{C}_{3}$; bottom: fourfold substitution in a double vacancy, $\mathrm{Ge}_{-} \mathrm{C}_{4}$ ). The raw MAADF/STEM images have been averaged from 7 and 40 frames, and further double Gaussian filtered. Quantitative STEM simulation using our experimental parameters reproduces in both cases the high intensity of the Ge impurity. DFT simulations show that while the Ge- $\mathrm{C}_{3}$ impurity buckles out of the graphene plane, the $\mathrm{Ge}-\mathrm{C}_{4}$ site is flat. The fields of view are $\sim 1 \times 1 \mathrm{~nm}^{2}$.

significant structural rearrangement expected around the $\mathrm{Ge}-\mathrm{C}_{3}$ site ${ }^{27}$ with the Ge atom buckling $1.90 \AA$ out of the graphene plane as shown in the top and side views of the relaxed model structure (Fig. 3).

Finally, inspired by recent electron-beam manipulation of impurity atoms in graphene, ${ }^{23,39-41}$ we attempted to move the Ge atoms by iteratively placing the electron beam on one $\mathrm{C}$ neighbor for $10 \mathrm{~s}$ between acquiring images. A total of 24 such irradiations were attempted on several different impurities, without success. DFT/MD simulations (described below) confirmed the reason: the beam-induced out-of-plane dynamics of the $\mathrm{C}$ atom, all the way up to its knock-on threshold of $15.75 \mathrm{eV}$, are unable to trigger the bond inversion mechanism for Ge. This stands in contrast with $\mathrm{B},{ }^{39} \mathrm{~N},{ }^{39} \mathrm{Si},{ }^{42} \mathrm{P},{ }^{41} \mathrm{Al}^{41}$ and, interestingly, even $\mathrm{Fe},{ }^{43}$ all of which have either been predicted or observed to move within the lattice. For Ge itself, the displacement threshold energy is $11.75 \mathrm{eV}$, almost an order of magnitude more than what a 60-keV electron can transfer to such a heavy nucleus. However, we observed a curious effect: 

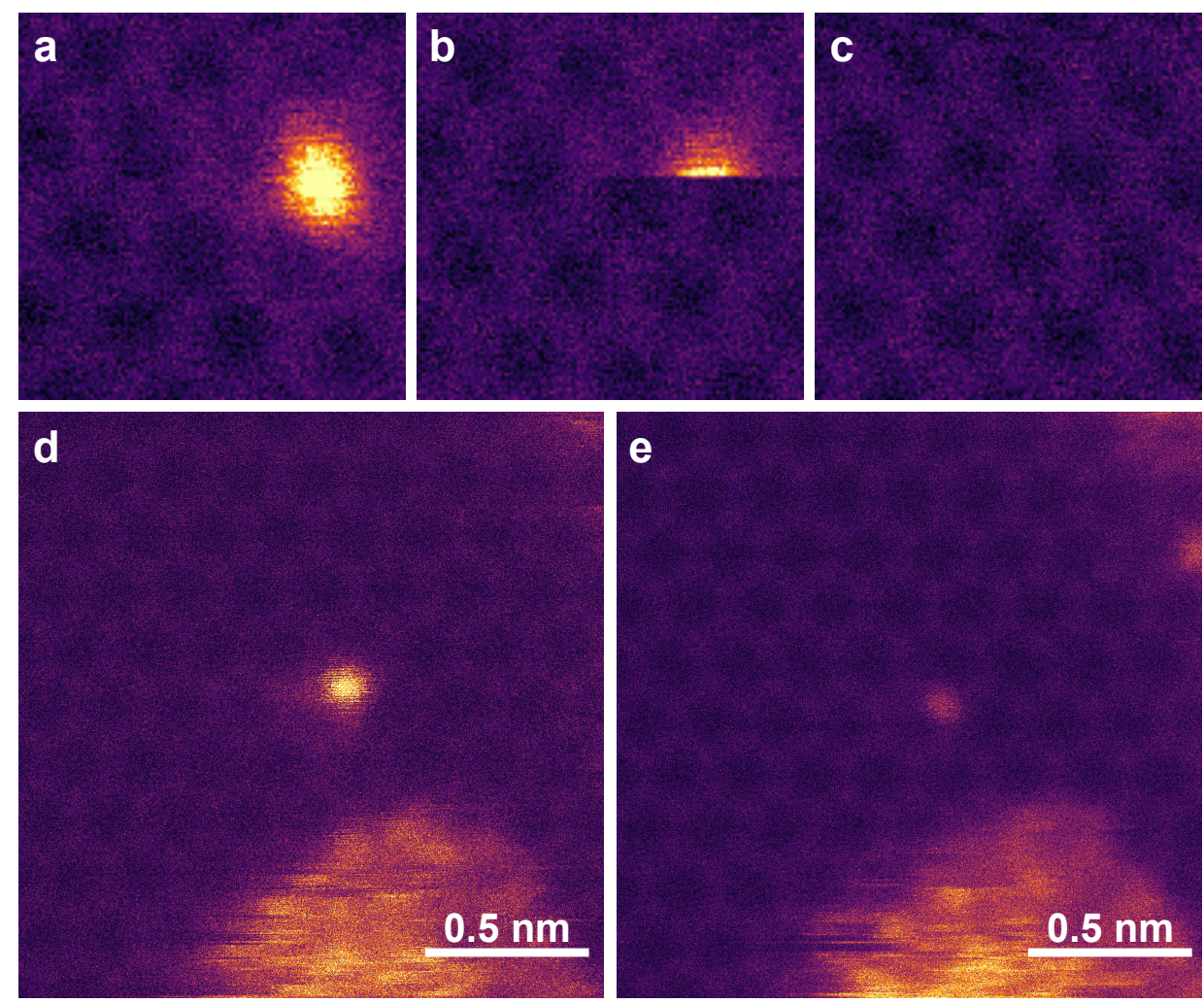

Figure 4: Ge replacement under electron irradiation (MAADF/STEM). a-c) Three consecutive raw images of a single Ge impurity being replaced by $\mathrm{C}$ during the second frame ( $\sim 4$ s per frame). d-e) Replacement of another Ge impurity by Si.

several Ge impurities got replaced by C (Fig. 4a-c) or, in one case, Si (Fig. 4d-e). Presumably the source of these atoms is the ubiquitous contamination, but the electron beam must be involved in the mechanism ${ }^{41}$ (since otherwise we would not be able to find any Ge in the lattice).

DFT-based molecular dynamics simulations were performed to analyze the energetics and atomic scale details of the implantation process, as well as for finding the displacement threshold energies for both Ge and its C neighbors and to study the possibility of the bond inversion mechanism. As mentioned above, we found that the mechanism, originally discovered for $\mathrm{Si}^{42}{ }^{42}$ does not work for Ge. This seems to be mainly due to the greater mass of Ge: during the out-of-plane trajectory of the ejecting $\mathrm{C}$ atom, the Ge does not move sufficiently fast to relax into the resulting vacancy. As a result, the $\mathrm{C}$ atom does cannot pass onto the other side of the impurity, and thus the Ge does not move in the lattice. 
Turning then to implantation, the successful incorporation of a heteroatom into graphene lattice by ion irradiation two conditions need to be satisfied: i) the energy transferred from the incident ion to carbon atom during collision is large enough to knock out carbon(s), and ii) the remaining kinetic energy of the ion after collision is low enough so that it stays in the structure. The energy transferred from the incident ion to a lattice atom is maximum for the case of a head-on collision, and therefore such impacts are most efficient in satisfying the above conditions.

We observed a number of outcomes depending on the kinetic energy of the incident Ge ion. Ions with energies below $20 \mathrm{eV}$ typically cannot induce breaking of the $\mathrm{C}-\mathrm{C}$ bonds and thus remain on the graphene surface as adatoms. For ion energies between 20-22 eV, Ge substitutes carbon atom in the lattice; however, the displaced carbon is not sputtered but remains bound to the system. All our simulations show that such configurations relax spontaneously to a structure consisting of non-defective graphene with a Ge adatom (although this may not be the case in experiments at room temperature). For Ge energies of $23-25 \mathrm{eV}$, a Ge-C dimer is formed that flies away from the graphene layer, in which a monovacancy defect is formed. Finally, at $26 \mathrm{eV}$ Ge is directly implanted into graphene structure while the $\mathrm{C}$ atom is sputtered. According to our calculations, direct implantation occurs for Ge kinetic energies ranging from 26 to $42 \mathrm{eV}$, while ions with higher energy penetrate through the graphene layer.

We find that in a head-on collision, an incident Ge ion transfers about $49 \%$ of its initial kinetic energy to an isolated carbon atom. The maximum kinetic energy of the impacted $\mathrm{C}$ atom in graphene is slightly less, $10.7 \mathrm{eV}$ (41\%) for an ion energy of $26 \mathrm{eV}$ (Fig. 5). This is significantly lower than the threshold energy for displacement of carbon in graphene, calculated with our method as $22.3 \mathrm{eV}$. Evidently, interactions between the incident Ge ion and $\mathrm{C}$ atoms in graphene significantly lower the displacement threshold energy of carbon.

To obtain a more detailed picture of the process, we analyse the kinetic energies of the incident Ge ion $\left(T_{\mathrm{Ge}}\right)$, sputtered $\mathrm{C}$ atom $\left(T_{\mathrm{C}}\right)$, remaining $\mathrm{C}$ atoms in the graphene layer 
$\left(T_{\mathrm{gr}}\right)$ and the total kinetic energy of the system $\left(T_{\mathrm{tot}}\right)$ at each time step of the simulation for an initial ion energy of $26 \mathrm{eV}$ (Fig. 5). At the beginning of the simulation, the total kinetic energy of the system equals that of the Ge ion. It slightly increases when the ion approaches graphene, with a maximum value of $27.8 \mathrm{eV}$, and starts to decrease quickly when the separation between the ion and graphene plane becomes less than $2.2 \AA$. The shortest Ge-C distance was found to be $1.53 \AA$. The energy transfer between the incident ion and the carbon atom occurs over tens of fs. When its kinetic energy reaches its maximum value of $10.7 \mathrm{eV}$, the $\mathrm{C}$ is already displaced from graphene plane (Fig. 5c) and, therefore, the maximum of $T_{\mathrm{C}}$ can be lower than the actual amount of the transferred energy. Subsequently, the kinetic energy of the sputtered $\mathrm{C}$ atom drops quickly by about $6 \mathrm{eV}$ and then gradually decreases to a constant value of $0.92 \mathrm{eV}$.

Interestingly, in simulations with higher energies, the long tail after the initial drop of $T_{\mathrm{C}}$ starts to disappear. For example, for an initial Ge energy of $40 \mathrm{eV}, T_{\mathrm{C}}$ becomes constant within $40 \mathrm{fs}$ and decreases by only $5.7 \mathrm{eV}$ from the maximum. This suggests that the $\mathrm{C}$ needs a kinetic energy of only about $5.7 \mathrm{eV}$ to overcome the interaction with the graphene lattice after a head-on collision with Ge. The long tail in $T_{\mathrm{C}}$ at low ion energies occurs mainly because of the interaction of $\mathrm{C}$ with the Ge ion, which can be considerable when both atoms are in close proximity and on the same side of graphene plane. At high values for the initial energies of the incident ion, the knocked-out $\mathrm{C}$ atom quickly moves away from graphene before the ion passes through the layer and therefore the tail in $T_{\mathrm{C}}$ disappears.

Coming back to the example shown in Fig. 5, the Ge ion continues to move in the same direction after the collision and the amount of kinetic energy transferred to $\mathrm{C}$ atoms in the lattice increases. A minimum in $T_{\mathrm{Ge}}$ at around $100 \mathrm{fs}$ corresponds to the point when the ion passes through the graphene layer. The subsequent increase in $T_{\mathrm{Ge}}$ occurs partially because the ion is moving towards a more energetically favourable configuration (Fig. 5d) and partially due to the interaction with the sputtered $\mathrm{C}$ atom. The energy increase is significantly smaller in simulations for higher ion energies, confirming that at low ion energies 

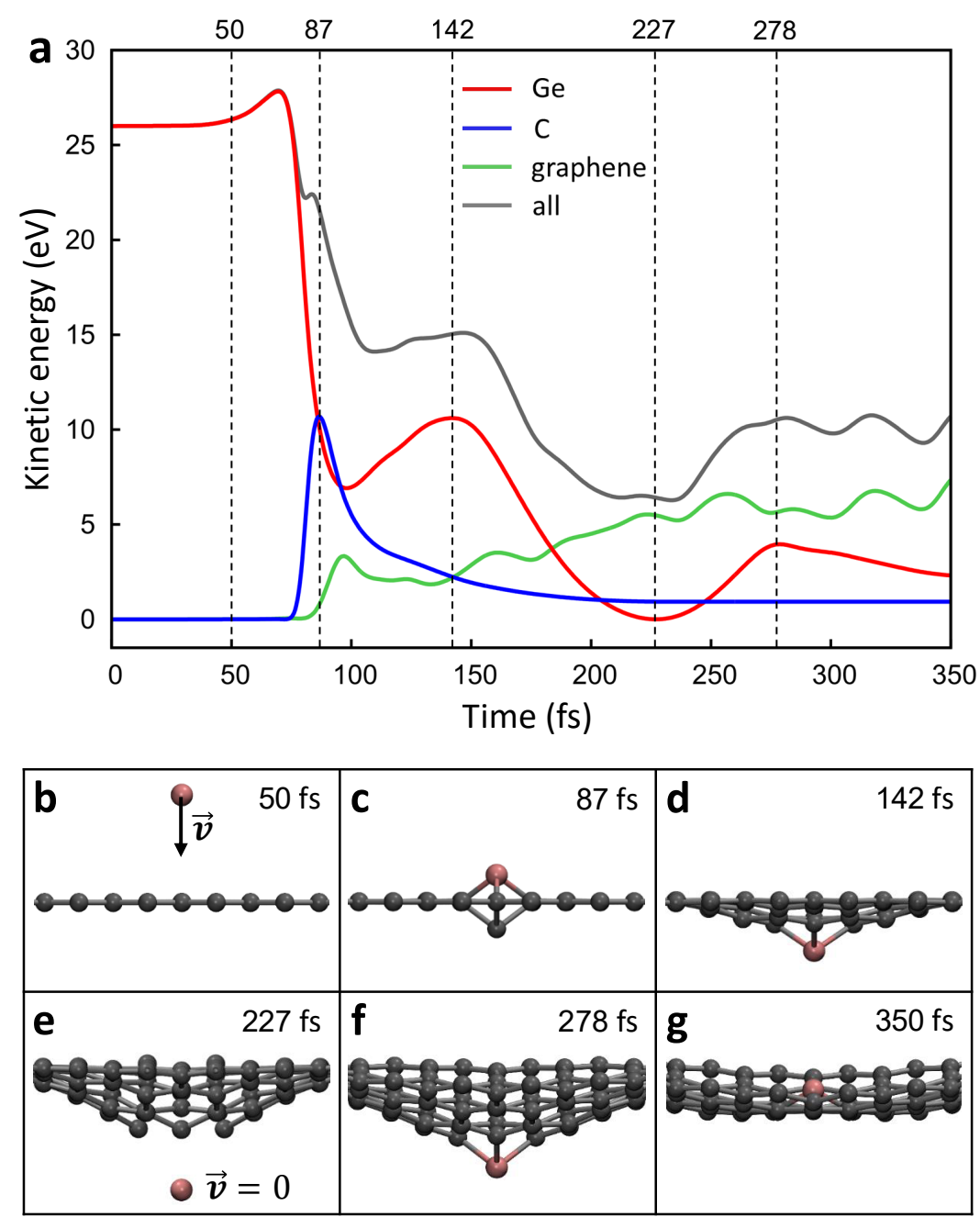

Figure 5: Energetics and atomic scale details of the implantation process. (a) Kinetic energies of the incident Ge ion (shown in red colour), sputtered $\mathrm{C}$ atom (blue), all remaining $\mathrm{C}$ atoms in the graphene layer (green) and total kinetic energy of the system (grey) as a function of time, obtained from the DFT/MD calculations for an initial ion energy of $26 \mathrm{eV}$. b-g) Atomic configurations that correspond to time steps marked in (a) by vertical dashed lines. The Ge atom is shown with pink colour, while carbon atoms are shown in grey.

the Ge-C interaction plays an important role. The kinetic energy of the ion then goes to zero while $T_{\text {gr }}$ gradually increases. Fig. 5e shows the configuration at which $T_{\mathrm{Ge}}$ equals zero. Although the ion has travelled a significant distance (about $4.3 \AA$ ) past the plane of graphene, the interaction with the highly buckled lattice is strong enough to stop and reverse the motion of the ion. The distance between the Ge and the undercoordinated $\mathrm{C}$ atoms at this time step is about $2.9 \AA$. Moving backwards, the ion gains enough kinetic 
energy to pass again through the graphene layer (Fig. 5f-g), before it finally stops, bound to the lattice. Further atomic motions in the system are relatively slow and do not involve significant structural rearrangements.

Finally, we should address the success of experimental implantation at energies below those predicted by our modeling. Considering the finite precision of the experimental ion energy, possible variation in the local bias potential, and the sources of inaccuracy in the simulations (including the approximation of exchange and correlation, the neglect of spin, and any basis set superposition error), as well as the simulated penetration of Ge into the lattice already between $20-22 \mathrm{eV}$, the modeling is actually surprisingly accurate. A statistical comparison of different experimental ion energies, as well as simulations covering the entire impact parameter space, would be required to make more precise direct comparisons.

\section{Conclusions}

We have implanted thus far the heaviest directly observed graphene impurity, and shown that despite its size, germanium can substitute a single atom by bonding to three carbon neighbors. This demonstrates that elements from the fourth period may be incorporated into a graphitic lattice, suggesting that substitutions from groups 3-13 with possible applications including single-atom catalysis and magnetism may also be possible. Our first principles molecular dynamics simulations further reveal that due to a strong chemical interaction between the incoming Ge ion and the neighbours of the displaced $\mathrm{C}$ atom, implantation can be carried out at an energy below the displacement threshold for graphene. As in earlier works, sample contamination remains an issue, and preventing it during implantation is an important target for improving sample quality in future experiments. Nevertheless, ion implantation continues to progress towards its promise as a scalable and precise technique for controllably doping low-dimensional materials. 


\section{Methods}

Low-energy ${ }^{74} \mathrm{Ge}^{+}$ions were implanted at the HZDR ion beam center into commercially available monolayer graphene supported on Au TEM grids (Quantifoil(R) R 2/4, Graphenea). We used a mass-selected, twofold electrostatic raster-scanned ion implantation system (Danfysik A/S, Denmark, Model 1050), providing ion energies down to $100 \mathrm{eV}$. Neutralized ions were eliminated by deflecting the ${ }^{74} \mathrm{Ge}^{+}$ion beam and then decelerating it towards the target. To reduce energies down to $15 \mathrm{eV}$, a bias voltage was set to the sample holder by an adjustable anode potential. The samples were irradiated in a $9 \times 10^{-7}$ mbar vacuum at room temperature with a fluence of $1 \times 10^{14} \mathrm{~cm}^{-2}$ (estimated with multiple Faraday cups). We chose ion energies of 15, 20 and $25 \mathrm{eV}$ to minimize irradiation-induced damage. The amount of energy that can be transferred to a carbon atom due to a collision with such ions is below the experimentally estimated displacement threshold energy $(21.14 \mathrm{eV})$ of graphene. ${ }^{44}$ Lower energies than expected are sufficient due to a strong chemical effect between the incoming Ge ion and the structure around the $\mathrm{C}$ atom being displaced. In an effort to reduce contamination, ${ }^{45}$ a $445 \mathrm{~nm}$ laser diode was aimed at the sample through a viewport of the vacuum chamber both for $2 \mathrm{~min}$ before implantation (nominal laser power $480 \mathrm{~mW}$ ) and during the implantation $(240 \mathrm{~mW})$.

The samples were imaged in near ultra-high vacuum $\left(<10^{-9}\right.$ mbar $)$ in an aberrationcorrected Nion UltraSTEM100 scanning transmission electron microscope ${ }^{46}$ operated at an acceleration voltage of $60 \mathrm{kV}$, well below the knock-on damage threshold of graphene. ${ }^{44}$ The beam current was around $50 \mathrm{pA}$, the beam convergence semi-angle was $30 \mathrm{mrad}$, and angular range was 60-200 mrad for MAADF and 80-300 $\mathrm{mrad}$ for HAADF. Some images were processed using a double Gaussian filtering procedure ${ }^{47}$ and all colored with the ImageJ lookup table "mpl-magma" to highlight relevant details. Low-loss EELS was recorded in the same instrument using a Gatan PEELS 666 spectrometer retrofitted with an Andor iXon 897

electron-multiplying charge-coupled device (EMCCD) camera. ${ }^{24}$ The energy dispersion was $0.1 \mathrm{eV} /$ pixel (with an instrumental broadening of $\sim 0.4 \mathrm{eV}$ ) and the EELS collection semian- 
gle was 35 mrad. Additionally, for greater sensitivity for Ge, we collected energy-dispersive x-ray spectra in a Philips CM200 TEM instrument operated at $80 \mathrm{kV}$.

To model the Ge substitution, we replaced one $\mathrm{C}$ atom in a $7 \times 4$ orthorhombic supercell of graphene (112 atoms in total), and relaxed its structure via DFT using the GPAW package ${ }^{48}$ (PBE functional, ${ }^{49} 0.16 \AA$ grid spacing, $5 \times 5 \times 1$ Monkhorst-Pack k-points ${ }^{50}$ ). The structure was used as input for a quantitative STEM simulation using the PyQSTEM interface ${ }^{51}$ to the QSTEM software package, ${ }^{52}$ with scattering potentials generated from the independent atom model. ${ }^{53}$ The same software package was used to find the displacement threshold energies for $\mathrm{C}$ and $\mathrm{Ge}$, as described previously ${ }^{44}$ (with a $0.2 \AA$ grid spacing and 0.1 fs timestep for the Velocity-Verlet dynamics).

The implantation of Ge was simulated using DFT-based molecular dynamics (MD) as implemented in the SIESTA code. ${ }^{54}$ We used Troullier-Martins norm-conserving pseudopotentials, ${ }^{55}$ the PBE functional, and a double- $\zeta$ polarized basis set. The charge density was represented on a real-space grid with an energy cutoff of 300 Ry. For these simulations, graphene was modeled using orthorhombic supercells consisting of 160 carbon atoms. A vacuum layer of $20 \AA$ was included in the direction normal to the graphene plane and the Brillouin zone was sampled using $3 \times 3 \times 1 \mathrm{k}$-points. To account for dispersion interactions, the Grimme semiempirical potential ${ }^{56}$ was used. The time step in MD calculations was set to $0.5 \mathrm{fs}$ and the initial kinetic energy of the Ge was varied at $1 \mathrm{eV}$ intervals. Although we call them ions for convenience, note that the projectiles in the simulations are neutral atoms.

\section{Acknowledgement}

We thank Sarah Haigh for additional characterization. M. Tripathi and T. Susi acknowledge funding by the Austrian Science Fund (FWF) through project P 28322-N36 and J. Kotakoski through project I3181-N36. J. Kotakoski was further supported by the Wiener Wissenschafts-Forschungs- und Technologiefonds (WWTF) through project MA14-009. A. 
Markevich and E. Besley acknowledge funding from the European Research Council under the European Union's Seventh Framework Programme (FP7) / ERC grant agreement № 307755-FIN. We thank the technical staff of the ion beam center facility of HZDR Dresden for providing us with support and facilities for the ion implantation experiments. DFT-based molecular dynamics simulations were performed using the high performance computing facility at the University of Nottingham, and further simulations at the HPC Midlands Plus Centre and the Vienna Scientific Cluster (VSC).

\section{References}

1. Novoselov, K. S.; Geim, A. K.; Morozov, S. V.; Jiang, D.; Zhang, Y.; Dubonos, S. V.; Grigorieva, I. V.; Firsov, A. A. Electric Field Effect in Atomically Thin Carbon Films. Science 2004, 306, 666-669.

2. Balandin, A. A.; Ghosh, S.; Bao, W.; Calizo, I.; Teweldebrhan, D.; Miao, F.; Lau, C. N. Superior Thermal Conductivity of Single-Layer Graphene. Nano Lett. 2008, 8, 902-907.

3. Lee, C.; Wei, X.; Kysar, J. W.; Hone, J. Measurement of the Elastic Properties and Intrinsic Strength of Monolayer Graphene. Science 2008, 321, 385-388.

4. Bolotin, K.; Sikes, K.; Jiang, Z.; Klima, M.; Fudenberg, G.; Hone, J.; Kim, P.; Stormer, H. Ultrahigh Electron Mobility in Suspended Graphene. Solid State Commun. 2008, $146,351-355$.

5. Bae, S.; Kim, H.; Lee, Y.; Xu, X.; Park, J.-S.; Zheng, Y.; Balakrishnan, J.; Lei, T.; Ri Kim, H.; Song, Y. I.; Kim, Y.-J.; Kim, K. S.; Özyilmaz, B.; Ahn, J.-H.; Hong, B. H.; Iijima, S. Roll-to-Roll Production of 30-inch Graphene Films for Transparent Electrodes. Nat. Nanotechnol. 2010, 5, 574 .

6. Xu, X.; Zhang, Z.; Qiu, L.; Zhuang, J.; Zhang, L.; Wang, H.; Liao, C.; Song, H.; Qiao, R.; Gao, P.; Hu, Z.; Liao, L.; Liao, Z.; Yu, D.; Wang, E.; Ding, F.; Peng, H.; 
Liu, K. Ultrafast Growth of Single-Crystal Graphene Assisted by a Continuous Oxygen Supply. Nat. Nanotechnol. 2016, 11, 930.

7. Stankovich, S.; Dikin, D. A.; Dommett, G. H. B.; Kohlhaas, K. M.; Zimney, E. J.; Stach, E. A.; Piner, R. D.; Nguyen, S. T.; Ruoff, R. S. Graphene-Based Composite Materials. Nature 2006, 442, 282-6.

8. Bonaccorso, F.; Sun, Z.; Hasan, T.; Ferrari, A. C. Graphene Photonics and Optoelectronics. Nat. Photon. 2010, 4, 611-622.

9. Guo, B.; Liu, Q.; Chen, E.; Zhu, H.; Fang, L.; Gong, J. R. Controllable N-Doping of Graphene. Nano Lett. 2010, 10, 4975-4980.

10. Banhart, F.; Kotakoski, J.; Krasheninnikov, A. V. Structural Defects in Graphene. ACS Nano 2011, 5, 26-41.

11. Ito, Y.; Christodoulou, C.; Nardi, M. V.; Koch, N.; Sachdev, H.; Müllen, K. Chemical Vapor Deposition of N-Doped Graphene and Carbon Films: The Role of Precursors and Gas Phase. ACS Nano 2014, 8, 3337-3346.

12. Persaud, A.; Park, S. J.; Liddle, J. A.; Rangelow, I. W.; Bokor, J.; Keller, R.; Allen, F. I.; Schneider, D. H.; Schenkel, T. Quantum Computer Development with Single Ion Implantation. Quantum Inf. Process. 2004, 3, 233-245.

13. Åhlgren, E. H.; Kotakoski, J.; Krasheninnikov, A. V. Atomistic Simulations of the Implantation of Low-Energy Boron and Nitrogen Ions into Graphene. Phys. Rev. B 2011, 83, 115424 .

14. Lehtinen, O.; Vats, N.; Algara-Siller, G.; Knyrim, P.; Kaiser, U. Implantation and Atomic-Scale Investigation of Self-Interstitials in Graphene. Nano Lett. 2015, 15, 235241. 
15. Kotakoski, J.; Krasheninnikov, A. V.; Ma, Y.; Foster, A. S.; Nordlund, K.; Nieminen, R. M. B and N Ion Implantation into Carbon Nanotubes: Insight from Atomistic Simulations. Phys. Rev. B 2005, 71, 205408.

16. Xu, F.; Minniti, M.; Giallombardo, C.; Cupolillo, A.; Barone, P.; Oliva, A.; Papagno, L. Nitrogen Ion Implantation in Single Wall Carbon Canotubes. Surf. Sci. 2007, 601, 28192822 .

17. Bangert, U.; Pierce, W.; Kepaptsoglou, D. M.; Ramasse, Q.; Zan, R.; Gass, M. H.; Van den Berg, J. A.; Boothroyd, C. B.; Amani, J.; Hofsss, H. Ion Implantation of Graphene-Toward IC Compatible Technologies. Nano Lett. 2013, 13, 4902-4907.

18. Willke, P.; Amani, J. A.; Sinterhauf, A.; Thakur, S.; Kotzott, T.; Druga, T.; Weikert, S.; Maiti, K.; Hofsss, H.; Wenderoth, M. Doping of Graphene by Low-Energy Ion Beam Implantation: Structural, Electronic, and Transport Properties. Nano Lett. 2015, 15, $5110-5115$.

19. Bangert, U.; Stewart, A.; OConnell, E.; Courtney, E.; Ramasse, Q.; Kepaptsoglou, D.; Hofsss, H.; Amani, J.; Tu, J.-S.; Kardynal, B. Ion-Beam Modification of 2-D Materials - Single Implant Atom Analysis via Annular Dark-Field Electron Microscopy. Ultramicroscopy 2017, 176, $31-36$.

20. Kepaptsoglou, D.; Hardcastle, T. P.; Seabourne, C. R.; Bangert, U.; Zan, R.; Amani, J. A.; Hofsss, H.; Nicholls, R. J.; Brydson, R. M. D.; Scott, A. J.; Ramasse, Q. M. Electronic Structure Modification of Ion Implanted Graphene: The Spectroscopic Signatures of p- and n-Type Doping. ACS Nano 2015, 9, 11398-11407.

21. Zhou, W.; Kapetanakis, M. D.; Prange, M. P.; Pantelides, S. T.; Pennycook, S. J.; Idrobo, J.-C. Direct Determination of the Chemical Bonding of Individual Impurities in Graphene. Phys. Rev. Lett. 2012, 109, 206803. 
22. Ramasse, Q. M.; Seabourne, C. R.; Kepaptsoglou, D.-M.; Zan, R.; Bangert, U.; Scott, A. J. Probing the Bonding and Electronic Structure of Single Atom Dopants in Graphene with Electron Energy Loss Spectroscopy. Nano Lett. 2013, 13, 4989-4995.

23. Susi, T.; Meyer, J.; Kotakoski, J. Manipulating Low-Dimensional Materials Down to the Level of Single Atoms with Electron Irradiation. Ultramicroscopy 2017, 180, 163-172.

24. Susi, T.; Hardcastle, T. P.; Hofsäss, H.; Mittelberger, A.; Pennycook, T. J.; Mangler, C.; Drummond-Brydson, R.; Scott, A. J.; Meyer, J. C.; Kotakoski, J. Single-Atom Spectroscopy of Phosphorus Dopants Implanted into Graphene. 2D Mater. 2017, 4, 021013.

25. Denis, P. A.; Iribarne, F. Dual Doped Monolayer and Bilayer Graphene: The Case of 4p and 2p Elements. Chem. Phys. Lett. 2016, 658, $152-157$.

26. She, X.; Li, Q.; Ma, N.; Sun, J.; Jing, D.; Chen, C.; Yang, L.; Yang, D. Creation of $\mathrm{Ge}-\mathrm{N}_{x}-\mathrm{C}_{y}$ Configures in Carbon Nanotubes: Origin of Enhanced Electrocatalytic Performance for Oxygen Reduction Reaction. ACS Appl. Mater. Interfaces 2016, 8, 10383-10391.

27. Ould Ne, M. L.; Abbassi, A.; El hachimi, A. G.; Benyoussef, A.; Ez-Zahraouy, H.; El Kenz, A. Electronic Optical, Properties and Widening Band Gap of Graphene with Ge Doping. Opt. Quantum Electron. 2017, 49, 218.

28. Nieman, R.; Aquino, A. J. A.; Hardcastle, T. P.; Kotakoski, J.; Susi, T.; Lischka, H. Structure and Electronic States of a Graphene Double Vacancy with an Embedded Si Dopant. J. Chem. Phys. 2017, 147, 194702.

29. Denis, P. A. Chemical Reactivity and Band-Gap Opening of Graphene Doped with Gallium, Germanium, Arsenic, and Selenium Atoms. ChemPhysChem 2014, 15, 39944000. 
30. Eberlein, T.; Bangert, U.; Nair, R. R.; Jones, R.; Gass, M.; Bleloch, A. L.; Novoselov, K. S.; Geim, A.; Briddon, P. R. Plasmon Spectroscopy of Free-Standing Graphene Films. Phys. Rev. B 2008, 77, 233406.

31. Krivanek, O. L.; Chisholm, M. F.; Nicolosi, V.; Pennycook, T. J.; Corbin, G. J.; Dellby, N.; Murfitt, M. F.; Own, C. S.; Szilagyi, Z. S.; Oxley, M. P.; Pantelides, S. T.; Pennycook, S. J. Atom-by-Atom Structural and Chemical Analysis by Annular DarkField Electron Microscopy. Nature 2010, 464, 571-574.

32. Zhou, W.; Lee, J.; Nanda, J.; Pantelides, S. T.; Pennycook, S. J.; Idrobo, J.-C. Atomically Localized Plasmon Enhancement in Monolayer Graphene. Nat. Nanotechnol. 2012, 7, 161-165.

33. Hage, F. S.; Hardcastle, T. P.; Gjerding, M. N.; Kepaptsoglou, D. M.; Seabourne, C. R.; Winther, K. T.; Zan, R.; Amani, J. A.; Hofsaess, H. C.; Bangert, U.; Thygesen, K. S.; Ramasse, Q. M. Local Plasmon Engineering in Doped Graphene. ACS Nano 2018, 12, $1837-1848$.

34. Gatan, Inc., EELS Atlas. 2018; http://www.eels.info/atlas.

35. Nguyen, P. D.; Kepaptsoglou, D. M.; Erni, R.; Ramasse, Q. M.; Olsen, A. Quantum Confinement of Volume Plasmons and Interband Transitions in Germanium Nanocrystals. Phys. Rev. B 2012, 86, 245316.

36. Krivanek, O. L.; Lovejoy, T. C.; Dellby, N.; Aoki, T.; Carpenter, R. W.; Rez, P.; Soignard, E.; Zhu, J.; Batson, P. E.; Lagos, M. J.; Egerton, R. F.; Crozier, P. A. Vibrational Spectroscopy in the Electron Microscope. Nature 2014, 514, 209-212.

37. Lovejoy, T. C.; Ramasse, Q. M.; Falke, M.; Kaeppel, A.; Terborg, R.; Zan, R.; Dellby, N.; Krivanek, O. L. Single Atom Identification by Energy Dispersive X-ray Spectroscopy. Appl. Phys. Lett. 2012, 100, 154101. 
38. Susi, T.; Skákalová, V.; Mittelberger, A.; Kotrusz, P.; Hulman, M.; Pennycook, T. J.; Mangler, C.; Kotakoski, J.; Meyer, J. C. Computational Insights and the Observation of SiC Nanograin Assembly: Towards 2D Silicon Carbide. Sci. Rep. 2017, 7, 4399.

39. Susi, T.; Kepaptsoglou, D.; Lin, Y.-C.; Ramasse, Q.; Meyer, J. C.; Suenaga, K.; Kotakoski, J. Towards Atomically Precise Manipulation of 2D Nanostructures in the Electron Microscope. 2D Mater. 2017, 4, 042004.

40. Dyck, O.; Kim, S.; Kalinin, S. V.; Jesse, S. Placing Single Atoms in Graphene with a Scanning Transmission Electron Microscope. Appl. Phys. Lett. 2017, 111, 113104.

41. Su, C.; Tripathi, M.; Yan, Q.-B.; Wang, Z.; Zhang, Z.; Basile, L.; Su, G.; Dong, M.; Kotakoski, J.; Kong, J.; Idrobo, J.-C.; Susi, T.; Li, J. Competing Dynamics of Single Phosphorus Dopant in Graphene with Electron Irradiation. 2018, arXiv:1803.01369.

42. Susi, T.; Kotakoski, J.; Kepaptsoglou, D.; Mangler, C.; Lovejoy, T. C.; Krivanek, O. L.; Zan, R.; Bangert, U.; Ayala, P.; Meyer, J. C.; Ramasse, Q. Silicon-Carbon Bond Inversions Driven by 60-keV Electrons in Graphene. Phys. Rev. Lett. 2014, 113, 115501.

43. Markevich, A. V.; Baldoni, M.; Warner, J. H.; Kirkland, A. I.; Besley, E. Dynamic Behavior of Single Fe Atoms Embedded in Graphene. J. Phys. Chem. C 2016, 120, $21998-22003$.

44. Susi, T.; Hofer, C.; Argentero, G.; Leuthner, G. T.; Pennycook, T. J.; Mangler, C.; Meyer, J. C.; Kotakoski, J. Isotope Analysis in the Transmission Electron Microscope. Nat. Commun. 2016, 7, 13040.

45. Tripathi, M.; Mittelberger, A.; Mustonen, K.; Mangler, C.; Kotakoski, J.; Meyer, J. C.; Susi, T. Cleaning Graphene: Comparing Heat Treatments in Air and in Vacuum. Phys. Status Solidi RRL 2017, 11, 1700124. 
46. Krivanek, O. L.; Corbin, G. J.; Dellby, N.; Elston, B. F.; Keyse, R. J.; Murfitt, M. F.; Own, C. S.; Szilagyi, Z. S.; Woodruff, J. W. An Electron Microscope for the AberrationCorrected Era. Ultramicroscopy 2008, 108, 179-195.

47. Krivanek, O. L.; Dellby, N.; Murfitt, M. F.; Chisholm, M. F.; Pennycook, T. J.; Suenaga, K.; Nicolosi, V. Gentle STEM: ADF Imaging and EELS at Low Primary Energies. Ultramicroscopy 2010, 110, $935-945$.

48. Enkovaara, J.; Rostgaard, C.; Mortensen, J. J.; Chen, J.; Dulak, M.; Ferrighi, L.; Gavnholt, J.; Glinsvad, C.; Haikola, V.; Hansen, H. A.; Kristoffersen, H. H.; Kuisma, M.; Larsen, A. H.; Lehtovaara, L.; Ljungberg, M.; Lopez-Acevedo, O.; Moses, P. G.; Ojanen, J.; Olsen, T.; Petzold, V. et al. Electronic Structure Calculations with GPAW: A Real-Space Implementation of the Projector Augmented-Wave Method. J. Phys.: Condens. Matter 2010, 22, 253202.

49. Perdew, J. P.; Burke, K.; Ernzerhof, M. Generalized Gradient Approximation Made Simple. Phys. Rev. Lett. 1996, 77, 3865-3868.

50. Monkhorst, H. J.; Pack, J. D. Special Points for Brillouin-Zone Integrations. Phys. Rev. $B$ 1976, 13, 5188-5192.

51. Susi, T.; Madsen, J.; Ludacka, U.; Jørgen Mortensen, J.; Pennycook, T. J.; Lee, Z.; Kotakoski, J.; Kaiser, U.; Meyer, J. C. Efficient First Principles Simulation of Electron Scattering Factors for Transmission Electron Microscopy. 2018, arXiv:1803.05338.

52. Koch, C. Determination of Core Structure Periodicity and Point Defect Density along Dislocations. Ph.D. thesis, Arizona State University, 2002.

53. Rez, D.; Rez, P.; Grant, I. Dirac-Fock Calculations of X-ray Scattering Factors and Contributions to the Mean Inner Potential for Electron Scattering. Acta Crystallogr. Sect. A: Found. Crystallogr. 1994, 50, 481-497. 
54. Soler, J. M.; Artacho, E.; Gale, J. D.; García, A.; Junquera, J.; Ordejón, P.; SánchezPortal, D. The SIESTA Method for Ab Initio Order-N Materials Simulation. J. Phys.: Condens. Matter 2002, 14, 2745.

55. Troullier, N.; Martins, J. L. Efficient Pseudopotentials for Plane-Wave Calculations. II. Operators for Fast Iterative Diagonalization. Phys. Rev. B 1991, 43, 8861-8869.

56. Grimme, S. Semiempirical GGA-Type Density Functional Constructed with a LongRange Dispersion Correction. J. Comput. Chem. 2006, 27, 1787-1799. 